\title{
Analysis in magneto crystalline anisotropy energy and intrinsic coercivity for body-centered cubic crystal lattices
}

\begin{abstract}
Magnetic diploe moment can be modeled in a similar manner to a loop of wire carrying current I. Energy stored in that dipole moment can be obtained by integrating the torque produced by that current carrying current loop. The summation of magnetic dipole moment over the volume $\Delta \mathrm{v}$ yields a new property of material called magnetization. The property of aligning domains within a permanent magnet itself with its internal field and in absence of external field is called spontaneous magnetization. In other words, a permanent magnet should sustain flux by virtue of its own internal field that requires spontaneous alignment of the magnetic dipole moments, or spontaneous magnetization. Magnetic materials are made in such a way that they have properties in one preferred axis that is easily possible using anisotropic materials because of their lattice structure. Magneto Crystalline anisotropy energy refers as the change in energy required to rotate the magnetic dipole $\mu \mathrm{m}$ by an angle $\phi$ that is required to rotate $\mu \mathrm{m}$ from a preferred axis $(\phi=0)$. Body centered cubic crystal lattice structure with six preferred direction of magnetization is depicted in this paper. ${ }^{1}$
\end{abstract}

Keywords: smagneto crystalline, energy, crystal lattices, magnetization, flux density, electromagnets
Volume 5 Issue 4 - 2018

Amrit Panthi, Bipin Lamichhane, Dhiraj Basnet, Anusha Lamichhane

Department of Electrical Engineering, Institute of Engineering (IOE), Tribhuvan University Kathmandu, Nepal

Correspondence: Amrit Panthi, Department of Electrical Engineering, Institute of Engineering (IOE), Pulchowk Campus, Tribhuvan University Kathmandu, Nepal,Tel +977 9840066285 , Email 07Ibel304@pcampus.edu.np

Received: July 12, 2018 | Published: August 13, 2018

\section{Energy stored in a dipole moment ${ }^{2}$}

The torque developed by a small area $\delta A$ depends upon the area of the strip and its magnetic flux density. ${ }^{3}$

$$
\delta T=i \delta A \operatorname{Bsin} \phi
$$

Integrating the equation 1

$$
\mathrm{T}=\mathrm{iAB} \sin \phi
$$

Current time's area in a magnetic circuit can be symbolized as the Magnetic Dipole Moment.

$$
\begin{gathered}
\mu_{m}=i A \\
T=\mu_{m} B \sin \phi
\end{gathered}
$$

The energy constituted within a dipole having torque ' $\mathrm{T}$ ' can be derived from the equation (3)

$$
\begin{gathered}
E=\int T . d \phi \\
E=-\mu_{o} \mu_{m} M \cos \phi
\end{gathered}
$$

The energy obtained from a magnetic dipole assuming it to be a current carrying loop is obtained as in equation (4).

\section{Magneto crystalline anisotropy}

Some of the materials itself has preferred directions for magnetic moments. These alignments of the magnetic dipole moments in the lattice are called magneto crystalline anisotropy. ${ }^{4}$ Equation (4) implies that the work done to rotate the $\mu \mathrm{m}$ with magnetization ' $\mathrm{M}$ '. This work done is minimum when $\mu \mathrm{m}$ and $\mathrm{M}$ are aligned to each other.

Equation (4) can be written as;

$$
E=-\mu_{o} \mu_{m} M\left(1-2 \sin ^{2} \frac{\phi}{2}\right)
$$

Magneto Crystalline Anisotropy Energy Ek can be defined as the additional energy required to rotate $\mu \mathrm{m}$ from a preferred axis $(\phi=0)$.

$$
E_{k}=2 \mu_{o} \mu_{m} M\left(\sin ^{2} \frac{\phi}{2}\right)
$$

There are six preferred direction of magnetization in a body centered cubic crystal lattice.

$$
\begin{aligned}
& {[0,0,1]-\text { Positive } z \text { direction }} \\
& {[0,1,0] \text { - Positive y direction }} \\
& {[1,0,0] \text { - Positive } x \text { direction }} \\
& {[0,0,-1] \text { - Negative } z \text { direction }} \\
& {[0,-1,0]-\text { Negative y direction }} \\
& {[-1,0,0]-\text { Negative } x \text { direction }}
\end{aligned}
$$

In order to increase the periodicity in equation (6), we modify the equation (6) as;

$$
E_{k}=2 \mu_{o} \mu_{m} M\left(\sin ^{2} 2 \phi\right)
$$

Plot for equation (7) is provided below;

Figure 1 shows the variation of Magneto Crystalline Anisotropy Energy ${ }^{6}$ with changing phi. Phi is represented in Figure 2. 


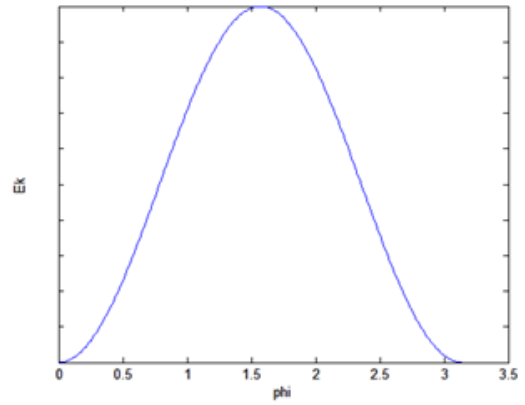

Figure I Magnetocrystalline anisotropy energy in a cubic crystal lattice structure.

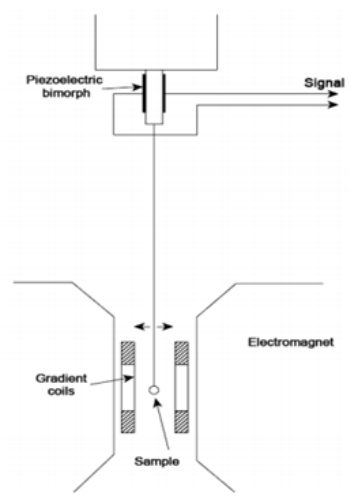

Figure 2 An illustration of magnetomenter.

Equation (7) can be represented as

$$
E_{k}=k \sin ^{2} 2 \phi
$$

Here, $\mathrm{k}$ is commonly described as a crystallographic constant that is experimentally identified using a tool called torque magnetometer. Magnetocrystalline Anisotropy tries to maintain the alignment of its domains whereas the external electromagnets ${ }^{5}$ try to oppose the anisotropy. These two forces create a torque that is measured by the magnetometer. The data from the device can be used to obtain the crystallographic constant of a material..$^{7-12}$ The action of two forces creating a net torgue is shown in the Figure 3.

Figure 3 Illustration of Magnetization force $(\mathrm{M})$ of the sample and the

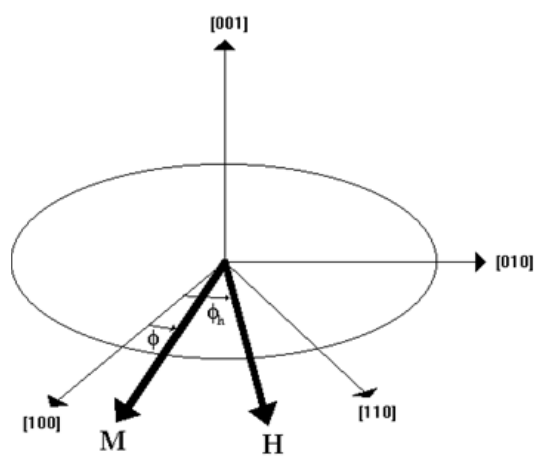

magnetizing field force of the electromagnet $(\mathrm{H})$.

Considering a bulk of iron sample that is already spontaneously magnetized in its posative $\mathrm{x}$ axis direction or simply towards $[1,0$, $0]$. But whenever a sufficient external magnetizing field is applied to the sample then all the magnetic moments would align along with the magnetizing field of the electromagnet. ${ }^{14-16}$

Assuming $\Phi$ to be the angle of saturated magnetic field ' $M$ ' of the sample with posative $\mathrm{x}$ axis and $\Phi \mathrm{h}$ be the angle of the magnetizing field ' $\mathrm{H}$ ' with posative $\mathrm{x}$ axis $[1,0,0]$.

The component of $\mathrm{M}$ that acts along the direction of the applied field $\mathrm{H}$ is given as

$$
M_{H}=M \cos \left(\phi_{h}-\phi\right)
$$

Here, $M_{H}$ is the component of saturated magnetic field $\mathrm{M}$ along the direction of applied field $\mathrm{H}$.

Applied field energy per unit volume after when $\mathrm{H}$ is at an angle of $\left(\phi_{h}-\phi\right)$ is given as

$$
E_{H}=-\mu_{0} M H \cos \left(\phi_{h}-\phi\right)
$$

Now, the total energy stored in the sample will be the sum of $E_{H}$ and $E_{k}$

So, adding equations (8) and (10), we get

$$
E=k \sin ^{2} 2 \phi-\mu_{0} M H \cos \left(\phi_{h}-\phi\right)
$$

Differentiating the equation (11) in order to obtain the minimum total energy.

$$
\frac{d E}{d \phi}=2 k_{1} \sin 4 \phi-\mu_{0} M H \sin \left(\phi_{h}-\phi\right)
$$

The intrinsic coercivity of a material is the value of $\mathrm{H}$ that causes M to suddenly reverse in opposite direction.

This intrinsic coercivity can be obtained by differentiating equation (12).

$$
\frac{d^{2} E}{d \phi^{2}}=8 k_{1} \cos 4 \phi-\mu_{0} M H \cos (\phi)
$$

For total reversal, the angle $\phi_{h}$ is $180^{\circ}$

At, $\phi=0, \frac{d^{2} E}{d \phi^{2}}=0$

Therefore, equation (13) is reduced to

$$
H_{c i}=\frac{8 k_{1}}{\mu_{0} M}
$$

$H_{c i}$ is the intrinsic coercivity.

Equation (14) provides a measure of the direct external demagnetization force that a sample can withstand.

\section{Conclusion}

The above plot in fig. 1 demonstrates that the unstable condition for $\mu \mathrm{m}$ lies at an angle of $\pi / 4$. Other elements used in permanent magnets may have complex lattice structure. This model for cubic crystal structure is of iron. ${ }^{17-19}$ This process helps to understand the basics of magnetic characteristics. Similarly, a brief knowledge can be extracted about the intrinsic coercivity or maximum demagnetization force that a sample can withstand.

\section{Acknowledgements}

None. 


\section{Conflict of interest}

The author declares that there is no conflict of interest involved in this study.

\section{References}

1. Hussain Shah SA. Vibrating Sample Magnetometery: Analysis and Construction. LUMS; 2013. p. 3-44.

2. Coillot C, Leroy P. Induction Magnetometers Principle, Modeling and Ways of Improvement. LPP; 2011. p. 1-64.

3. Held R, Kevin R. Construction of an Inexpensive Torque Magnetometer for Magnetic Thin Film Research. Stetson University.

4. Born M, Huang K. Dynamical Theory of Crystal Lattices. Oxford; 1954. p. 1-415.

5. Giselher Herzer. Modern soft magnets: Amorphous and nanocrystalline materials. Acta Materialia. 2013;61(3):718-734.

6. Kaiming Cai, Meiyin Yang, Hailang Ju, et al. Electric field control of deterministic current-induced magnetization switching switching in a hybrid ferromagnetic/ferroelectric structure. Cornell University Library; 2016. 15 p.

7. Landau, Lev Davidovich. Electron Motion in Crystal Lattices.

8. Maradudin AA. Some effect of point defects on the vibrations of crystal lattices. Reports on Progress in Physics. 2018;28.

9. Gorter EW. Saturation magnetization of some ferromagnetic oxides with hexagonal crystal structures. Journals \& magazines.
$1957 ; 104(5 S): 255-260$.

10. Mouhat F, Coudert FX. Necessary and sufficient elastic stability conditions in various crystal systems. Phys Rev. 2014.90(22).

11. Milstein F, Hill R. Theoritical properties of cubic crystals at arbitrary pressure-III Stability. Journal of the mechanics and physics of solids. 1979;27(3):255-279.

12. Cochran W. Crystal stability and the theory of ferroelectricity. American Physical Society. 1959;3(9):412.

13. Chung DH. The elastic anisotropy of crystals. Journal of Applied Physics. 2004;38(5).

14. Starostin NV, Feofilov PP. Magnetic circular anisotropy in crystals. Soviet Physics Uspekhi. 1969;12(2).

15. VAN Vleck JH. On the Anisotropy of cubic ferromagnetic crystals. Physical Review Journals Archive. 1937;55(11):1178.

16. Bozorth RM. Magnetostriction and crystal anisotropy of single crystals of hexagonal cobalt. Physical Review Journals Archive. 1954;96(2):311.

17. Spoor PS, Maynard JD, Kortan AR. Elastic isotropy and anisotropy in quasicrystalline and cubic AlCuLi. Phys Rev Lett. 1995;75(19):34623465 .

18. Itoh K, Zeng LZ, Nakamura E. Crystal structure of $\mathrm{BaTiO}_{3}$ in the cubic phase; 1985.

19. Kikuchi R, Sato H. Characteristics of super lattice formation in alloys of face centered cubic structure. Acta Metallurgica. 1974;22(9):10991112. 